\section{Reflexiones en torno a las prácticas integrales en la Universidad de la República}

Humberto Tommasino

htommasino@gmail.com

Felipe Stevenazzi

fstevenazzi@gmail.com

Docentes investigadores de la

Universidad de la República, Uruguay.
Integración de la docencia y la extensión /

Desafíos de gestión

RECEPCIÓN: 24/06/16

ACEPTACIÓN FINAL: 10/10/16

\section{Resumen}

El presente artículo se propone dialogar a partir de un conjunto de reflexiones que nos surgen luego del proceso de transformación de la Universidad de la República, Uruguay, en el marco de su Segunda Reforma, que tiene como uno de sus pilares la generación de prácticas integrales, encontrando en la extensión universitaria uno de sus motores. Se entiende la integralidad como la articulación de las funciones universitarias (enseñanza, extensión e investigación), interdisciplina y diálogo de saberes (científicos y populares). Se procurará abordar el proceso de resignificación de la extensión y el desafío de la integralidad y cómo esto puede enriquecer la formación universitaria y aportar a la transformación de lo que implica hacer universidad.

Palabras clave

- Integralidad

- Extensión universitaria

- Universidad de la República

\section{Resumo}

O artigo se propõe dialogar a partir de um conjunto de reflexões que surgem depois do processo de transformação da Universidade da República, Uruguai, no contexto da sua Segunda Reforma, que tem como um dos seus pilares a geração de práticas integrais, encontrando na extensão universitária um dos seus motores. Entende-se a integralidade como a articulação das funções universitárias (ensino, extensão e pesquisa), interdisciplinaridade e diálogo de saberes (científicos e populares). O artigo tentará abordar o processo de ressignificação da extensão e o desafio da integralidade e como isto pode enriquecer o ensino universitário e contribuir para a transformação do que significa fazer Universidade.

Palavras-chave

- Integralidade

- Extensão universitária

- Universidade de la República

Para citación de este artículo

Tommasino, H. y Stevenazzi, F. (2016). Reflexiones en torno a las prácticas integrales en la Universidad de la República. En Revista +E versión digital, (6), pp. 120-129. Santa Fe, Argentina: Ediciones UNL. 


\section{6}

para que se pueda desarrollar la

integralidad son necesarias una verdadera articulación y la transformación de las

funciones, donde la enseñanza (con sus

tiempos, lógicas y estrategias) no subordine a las demás funciones

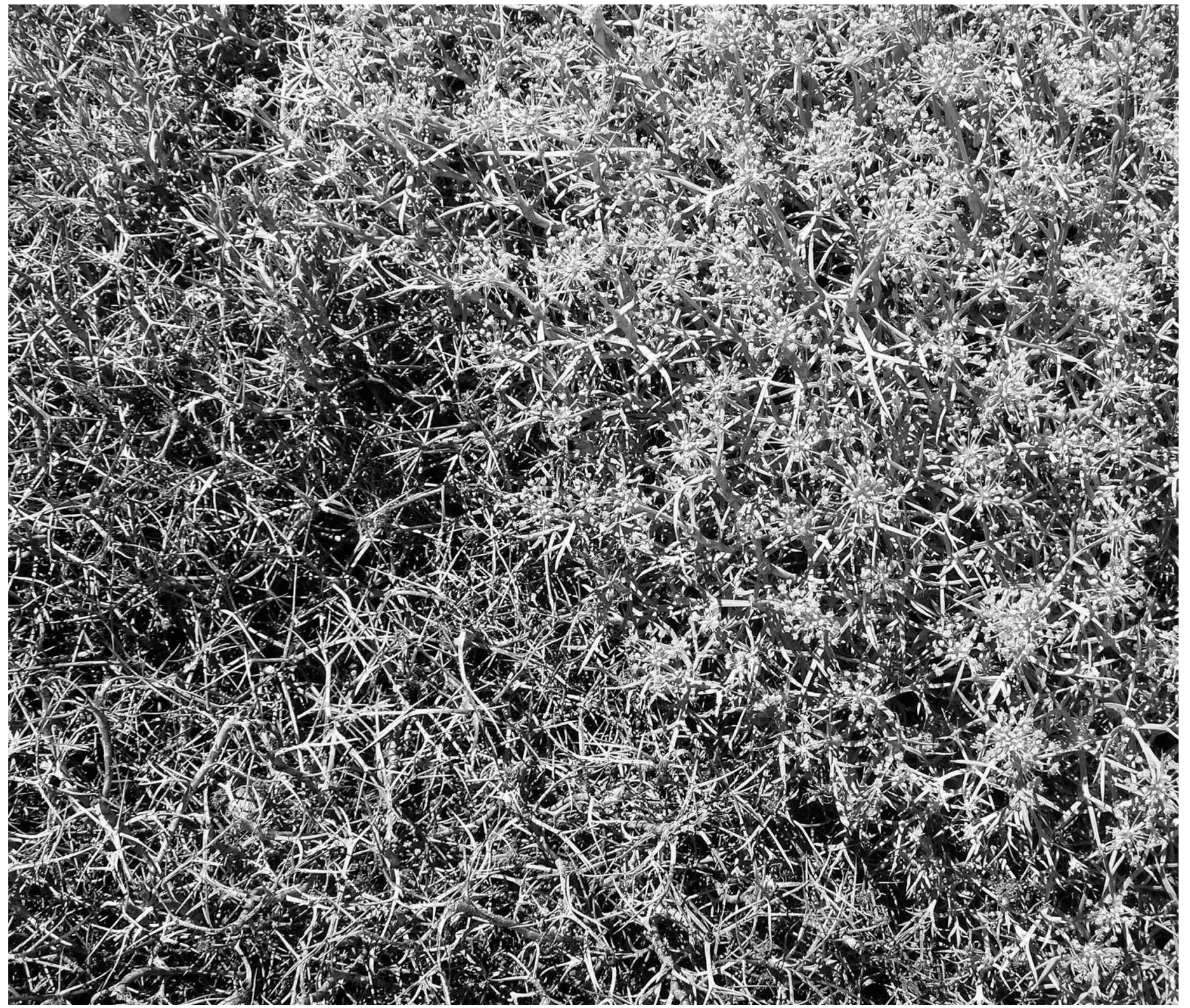




\section{Introducción}

Este trabajo se propone reflexionar sobre la extensión universitaria en el marco del desarrollo de las prácticas integrales en la Universidad de la República (UdelaR), Uruguay, mediante el abordaje del proceso de resignificación de la extensión y el desafío de la integralidad. En este sentido se propone revisar algunos aspectos teóricos referidos a la extensión y a la integralidad de las prácticas educativas incorporando algunas reflexiones y conclusiones que se realizan desde la implementación de los Espacios de Formación Integral (EFI) en la UdelaR.

En un contexto donde el conocimiento es cada vez más privatizado - sobre todo en Europa y Estados Unidos de América, hecho que se verifican también en algunos espacios de la educación terciaria latinoamericana, tornándose en vehículo para diversas formas de dominación social - la universidad no escapa a esta tendencia y quedan algunos espacios contratendenciales que debemos cuidar y desafiar para la transformación universitaria y social. Debe establecerse que, si bien ésta es la tendencia general, en algunos países del Cono Sur se han generado en los últimos diez años procesos importantes de expansión de la universidad pública. Este proceso es notorio en Brasil, Argentina y Uruguay, puesto que en estos países los gobiernos progresistas han aumentado los presupuestos para la educación y han creado varias universidades públicas y nuevos centros universitarios como ampliación de las universidades ya existentes, como es el caso de Uruguay, donde se constatan los dos fenómenos.

La extensión universitaria orientada al diálogo de saberes y a priorizar los sectores sociales más postergados ofrece una posibilidad y una forma de acercar la universidad al medio, y fundamentalmente de poner a los universitarios frente a nuevos desafíos y orientar una agenda de prioridades para transformar la enseñanza a través de diferentes interrelaciones con el conocimiento y con los otros.

Para que se pueda desarrollar la integralidad son necesarias una verdadera articulación y la transformación de las funciones, donde la enseñanza (con sus tiempos, lógicas y estrategias) no subordine a las demás funciones. Este es un riesgo latente dado que lo que mayormente hacemos en la universidad es enseñanza.

\section{Universidad e integralidad}

La UdelaR, poco a poco pero en forma constante, viene asumiendo el desafío de la integralidad, que se propone la transformación de ésta, a través de lo que en ella se realiza. En este sentido la transformación de las actividades de enseñanza son fundamentales, por un lado porque es la actividad que concentra la mayor parte del tiempo de docentes y estudiantes y por otro lado por la capacidad de formatear que tiene la enseñanza. Gran parte de lo que hacemos como universitarios tiene que ver con las experiencias positivas y negativas por las que transitamos en nuestra formación de grado.
Allí hay un punto central para comenzar la modificación de lo que se realiza, en la medida que las biografías escolares tienen un peso sustantivo en la formación de los profesionales y cómo esto luego conforma fundamentalmente a los que van a desarrollar la actividad docente, por lo tanto aquí hay una posibilidad de cambiar las prácticas estudiantiles, también de cara a esos futuros desarrollos. Es interesante analizar cómo recoge esta apuesta institucional la ordenanza de estudios de grado aprobada el 30 de agosto de 2011 en la sección de Orientaciones de enseñanza.

Esta ordenanza establece las orientaciones de los planes de estudio que se vienen modificando en varias facultades:

"b. Los procesos de enseñanza estarán integrados con las funciones universitarias de extensión y de investigación, en las cuales el estudiante será sujeto activo. En extensión, conocerá tempranamente en forma directa el medio específico en el cual se desarrolla el área de conocimiento elegida y participará en actividades de extensión debidamente integradas a la enseñanza. Por su parte en investigación, recibirá y analizará trabajos originales y sus resultados, y siempre que sea posible participará directamente en las actividades.

c. Las actividades de extensión y de investigación, adecuadamente orientadas y supervisadas por el grupo docente que corresponda, serán reconocidas en el sistema de créditos". (UdelaR, Ordenanza de Estudios de Grado, 2011:3)

La renovación de la enseñanza se propone también a partir de la curricularización de la extensión, tal como está planteada desde el título de la resolución que adopta el Consejo Directivo Central (CDC) "Hacia la reforma universitaria. La extensión en la renovación de la enseñanza. Espacios de formación integral". Queda explicitada de alguna forma la necesidad de renovar, transformar la enseñanza universitaria, en procura de superar antiguos modelos que vienen con la propia historia de la Universidad y que no necesariamente se adecuan a los sujetos que hoy la habitan y tampoco a las necesidades de la sociedad que sustenta esta Universidad.

El desarrollo de la extensión universitaria, tanto para estudiantes como docentes, puede facilitar algunas transformaciones en la propuesta pedagógica de la Universidad:

"la extensión entendida como un proceso dialógico y bidireccional redimensiona a la enseñanza, al aprendizaje y a la investigación. Si los procesos de enseñanza y aprendizaje se extienden fuera del aula, cuidando al mismo tiempo de mantener los mejores niveles académicos en las actividades desarrolladas, su enriquecimiento puede ser grande. Cuando las tareas se generan y operan en terreno, partiendo de los problemas que la sociedad tiene, intentando junto con ella encontrar alternativas, entonces el acto educativo se reconfigura y amplía”. (UdelaR, 2010:15) 
asumir el desarrollo de la integralidad

en todos sus términos implica la

transformación del modelo pedagógico

y de las relaciones que se establecen

con el conocimiento

Reconfigurar el acto educativo y transformar la enseñanza parecen formar parte de un acuerdo tácito, el agotamiento de un modelo pedagógico tiene que ver fundamentalmente con el cambio de los sujetos que entran para ser modelados, ahí comienza a producirse el desajuste. Al menos evidenciamos un cambio fundamental que tiene que ver con los tiempos que destinan los estudiantes a una determinada tarea; la formación requiere fundamentalmente invertir tiempo y resignar otras posibilidades. Como docentes debemos profundizar la búsqueda de estrategias para que el pasaje por la Universidad no se transforme en una colección de créditos y podamos desencadenar experiencias significativas que permitan a los estudiantes pensarse y reflexionar sobre su proceso de formación y los sentidos de ésta.

A la "intemperie del aula" (Tommasino y otros, 2010) exclaustrada, esta característica genera efectos en docentes y estudiantes, se reconfiguran las relaciones de saber-poder, permiten su circulación y colocan a los universitarios en el lugar de aprender de otros. En este contexto, la extensión puede transformarse en ese sentido, acercando a los estudiantes al contacto con las diferentes realidades sociales y a la conexión con lo que van aprendiendo, en relación con la sociedad y sus formas de entenderla. Este acercamiento necesariamente desafiará a estudiantes y docentes, colocándolos en el lugar de la incerteza y la búsqueda, actitudes fundamentales en cualquier proceso de formación. Esas realidades sociales se convierten en el principal instrumento didáctico para orientar la formación.

\section{Proceso de formación integral y puesta en práctica de otro modelo pedagógico}

Asumir el desarrollo de la integralidad en todos sus términos implica la transformación del modelo pedagógico y de las relaciones que se establecen con el conocimiento.

La articulación de funciones que supone la integralidad genera relaciones diferentes de las que pueden darse en un aula universitaria y en función de ello toda esa trama de relaciones se altera.

La relación educativa acontece en torno a una práctica que, como advierte Paulo Freire, es una dimensión necesaria de la práctica social, en su riqueza y complejidad, un fenómeno "exclusivamente humano" que a través de su estructuración se torna una práctica social educativa:

"El sujeto que enseñando aprende y el sujeto que aprendiendo enseña. Educador y educando. Objetos de conocimiento que han de ser enseñados y aprehendidos por los alumnos para que puedan aprenderlos. Contenidos. Objetivos, mediatos e inmediatos a los cuales se orienta la práctica educativa. Y por último métodos, técnicas y materiales didácticos coherentes con los objetivos y con la opción política”. (1996:77)

Una particularidad central de la relación educativa respecto de otras relaciones humanas es que está atravesada por el conocimiento y los actores están allí formando parte de esa relación para aprenderenseñar y viceversa, desde los distintos grados de apropiación de ese conocimiento. 
En los procesos de formación integral se modifica al menos esa asimetría entre docentes y estudiantes en cuanto al conocimiento, nos enfrentamos a la búsqueda y resolución de problemas, la extensión y la investigación nos ponen en contacto con lo no conocido, desafían las seguridades desde las cuales se estructuraba nuestro rol.

La extensión contribuye a los procesos de formación en la medida que habilita enseñanzas y aprendizajes en múltiples direcciones. Los universitarios son una parte de ese proceso y quizás los que pueden obtener el mayor provecho de ese relacionamiento con los actores no universitarios y generar nuevas relaciones con el conocimiento y nuevos conocimientos.

En una universidad que trabaja en la integralidad, la extensión funciona como un catalizador que permite articular y dar orientación a la producción de conocimiento en la medida en que habilita a estudiantes y docentes:

"la oportunidad de interrelacionar teoría y práctica así como la posibilidad de generar nuevas preguntas —no saberesdisparadores neurálgicos en la producción de nuevos conocimientos. A su vez, permite repensar y reformular el currículum de formación profesional y / o técnico en función de las nuevas exigencias y desafíos de la sociedad". (Bordoli, 2006:18)

Pensar un modelo pedagógico que recupere la integralidad como proceso de formación y transformación del quehacer universitario requiere que las funciones no se vean subordinadas entre sí. Proponemos entonces habilitar la búsqueda de un modelo pedagógico centrado en la formación como plantea Elsa Gatti (2000). Allí el énfasis no está puesto ni en el docente ni en el estudiante, sino en la relación entre ambos o, en sentido más amplio, en las relaciones pedagógicas (estudiante-estudiante, docente-estudiante, docente-docente, estudiante-docenteactores no universitarios) como propiciadoras de una dinámica de desarrollo personal. La no-directividad es una característica central de este modelo, lo que no implica un abandono de la responsabilidad docente. Por el contrario, asumir la no-directividad es habilitar el desarrollo de la autonomía en el otro, lo que necesita un proceso de acompañamiento que brinde seguridad de escoger determinadas opciones.

En este proceso de conquista de la autonomía, docentes y estudiantes asumen mayores grados de compromiso con su formación; ellos, en relación, se tornan los propios gestores de su formación, abandonan la pasividad y la concentración de poder en la conducción del proceso.
"Un grupo de formación que funcione con esta modalidad nodirectiva, se mueve entonces en dos dimensiones: como grupo de aprendizaje, que se propone y autorregula para la adquisición de determinados conocimientos y/o destrezas; y como grupo de formación psico-sociológica, que reflexiona sobre sí mismo y analiza las interacciones, los conflictos, los avances y retrocesos, los estancamientos, los logros, en fin: el proceso seguido individual y grupalmente". (Gatti, 2009:32)

Emprender este camino implica necesariamente abandonar las cómodas certezas y el espacio protegido del rol prefijado de docente y de estudiante, permitir que la realidad interrogue y desafíe. La extensión y la investigación nos desafían necesariamente a lo "por conocer"; desde diferentes lugares nos enfrentamos a lo desconocido en relación con otros, ya sea con los pares en la investigación, con los "otros" en la comunidad, y es necesariamente desde ese "nosotros" que se construye un nuevo conocimiento a ser desafiado dialécticamente.

Este proceso de búsqueda autónoma del conocimiento supone una definición política y también una transformación de las intervenciones que se desarrollan desde los procesos de extensión, al ser los actores sociales los sujetos promotores de las mismas. La universidad como centro de poder se ha relacionado históricamente con el medio en tanto sujeto proveedor de información y de temas a ser investigados. En cambio, desde la ética de la autonomía se rompe radicalmente con esta postura, se pregona justamente el conocimiento como posibilidad de conquistar la autonomía y no volverse conquistador.

\section{Desafíos pedagógicos y posibilidades para otro modelo}

La integralidad pone en situación de repensar la relación entre las funciones universitarias, plantea problemas nuevos, pone en relación a disciplinas y a su vez a éstas con los actores extrauniversitarios. Es una oportunidad privilegiada para ensayar relaciones con los otros que permitan replantear el lugar y las representaciones que en el pasado y en el presente se construyen y practican sobre y desde la universidad.

Construir una relación con los otros, la construcción de una relación que se articula en la diferencia, alguien diferente en el que puedo reconocerme, una diferencia que no enmascara una desigualdad. La universidad históricamente ha construido — salvo honrosas excepciones- esa relación a partir de una superioridad: participar de la distribución del conocimiento y de su apropiación no es más que un reflejo de la desigualdad que funda nuestra sociedad. 
Por lo tanto, la universidad se ubica y es ubicada en un sitial de privilegio. Pensar en otra forma de estructurar ese vínculo es fundamental para producir otras prácticas pedagógicas y otras relaciones.

En este sentido, la universidad debería ser un espacio para el cultivo de la autonomía, en la manera de establecer vínculos, posibilitar que éstos sean más horizontales y democráticos; estas prácticas puestas en relación con los actores extrauniversitarios pueden ser un instrumento pedagógico importante, una estrategia y una intencionalidad para construir con los otros.

Es interesante reflexionar sobre las formas en las que la autonomía tiene lugar en nuestras prácticas, sobre qué posibilidades de ejercicio y desarrollo de la autonomía contemplamos en nuestras propuestas.

Parece interesante detenerse sobre el lugar en el que ubicamos a los otros en nuestras propuestas, es decir, cómo quedan y dónde se pueden posicionar. Esto tiene directa relación con la autonomía y las intencionalidades pedagógicas que se ponen en juego. Generar espacios en los cuales contribuir a la reflexión conjunta sobre los problemas y diseñar salidas factibles a los mismos puede ser en muchos casos el aporte que la universidad plantee, pero de cómo lo haga depende que contribuya a la autonomía de esos otros o que, por el contrario, limite esa posibilidad.

\section{La ruptura del vínculo áulico exclusivo: el aprendizaje en el medio y el diálogo de saberes generado en la integralidad} Desde la educación popular se han destacado la potencia formativa del medio social y sus actores en situación. Freitas (en Pistrak, 2009) señala que:

"una pedagogía del medio es, antes que nada, una pedagogía crítica de su medio y que por lo tanto forma sujetos históricos (autoorganizados). Sería lógico pensar que este medio social no debería tener una función de anular la formación de tales sujetos históricos. De ahí el carácter dialéctico que asume la relación entre el ser en formación y su medio (natural o social). En este proceso el medio (y sus luchas) enseña y al mismo tiempo, es criticado como forma de desarrollar a ambos (el sujeto y su medio), lo que vale para cualquier formación social. (...) Los movimientos sociales también enseñan y no hay razón para que la escuela no se entrelace con ellos. Además: si la escuela es necesaria, pero insuficiente para producir muchas de las transformaciones sociales, su relación con los movimientos sociales permite potenciar su acción" (2009:92-93). ${ }^{1}$
Cuando se establecen vínculos dialógicos con los actores sociales presentes en el "medio", éstos asumen roles enseñantes, habilitan la circulación de saberes, plantean nuevos problemas reales, conjeturas sobre alternativas, ponen en evidencia respuestas ya experimentadas para resolver varias problemáticas, visualizan sus potencialidades y restricciones. Todas estas alternativas dialógicas con los actores sociales incorporan a la enseñanza y al aprendizaje una búsqueda más heurística que la que se plantea artificialmente en la sala de aula.

Los procesos de integralidad que incluyen la extensión crítica en los espacios educativos incorporan el diálogo de saberes que es definido por la integración de actores no universitarios que aprenden y enseñan junto a los estudiantes y docentes que también aprenden y enseñan. Se genera una vinculación entre saberes diferentes: los saberes científicos o académicos (en un sentido más amplio) y los saberes populares. La extensión crítica está vinculada con las concepciones de educación popular e investigación acción participación que emanan de estos procesos de lucha. A nivel de la universidad latinoamericana, también se pueden rastrear como procesos históricos fundantes de esta corriente algunas propuestas que se relacionan con el surgimiento de las universidades populares (Bralich, 2006). Deben reconocerse entonces como fuente inspiradora el movimiento de la educación popular latinoamericana y la impronta que han dado a este proceso algunos autores como Paulo Freire y Orlando Fals Borda, entre otros (Tommasino y Cano, 2016). Cuando revisamos los fines de la extensión crítica se pueden reconocer dos objetivos dialécticamente relacionados. El primero es el relativo a la formación de los universitarios y a la posibilidad de establecer procesos integrales que rompan con la exclusiva formación técnica y sin compromiso social de los graduados universitarios. La extensión concebida como un proceso crítico y dialógico debe contribuir a generar universitarios que tengan estas dos cualidades. Se debe trascender la formación exclusivamente técnica que genera la universidad concebida como una "fabrica de profesionales" y alcanzar procesos formativos integrales que generen universitarios comprometidos y solidarios con los más amplios sectores de las sociedades latinoamericanas. El segundo se refiere a que la universidad debe contribuir a los procesos de organización y autonomía de los sectores populares subalternos e intentar generar procesos de poder popular. Claro está, estos dos objetivos tienen una vinculación dialéctica y orgánica, no es posible avanzar en uno sin el avance del otro (Tommasino y Cano, 2016).

Es importante analizar cómo se ha entendido este vínculo entre saberes desde distintas concepciones epistemológicas para 
direccionarlo hacia procesos de horizontalidad y comunicación dialógica. Respecto de este último concepto, Freire entiende que:

"na comunicação, não há sujeitos passivos. Os sujeitos cointencionados ao objeto de seu pensar se comunicam seu conteúdo. O que caracteriza a comunicação enquanto êste comunicar comunicando-se, é que ela é diálogo, assim como o diálogo é comunicativo. Em relação dialógica-comunicativa, os sujeitos interlocutores se expressam, como já vimos, através de um mesmo sistema de signos linguísticos. É então indispensável ao ato comunicativo, para que êste seja eficiente, o acôrdo entre os sujeitos, recìprocamente comunicantes. Isto é, a expressão verbal de um dos sujeitos tem que ser percebida dentro de um quadro significativo comum ao outro sujeito". $(1985: 85)$

Enriquez y Figueroa (2014) identifican tres tesis diferentes a la hora de pensar la vinculación entre el saber popular y el saber académico-científico: una primera vinculada a la continuidad entre sentido común y saber científico, una de ruptura y otra vinculada a la ruptura de la ruptura y el reencuentro.

La primera propuesta entiende que el punto de partida del saber académico es el sentido común. Esta tesis es sostenida tanto por autores "neopositivistas" como Popper, como por autores que han aportado a la teoría crítica,, como Fals Borda. En el primer caso, Enriquez y Figueroa (2014) partiendo de Popper, nos dicen que éste entiende que el sentido común produce un conocimiento elemental de carácter lógico porque parte de una base racional, pero puede ser cuestionada porque efectúa elaboraciones imperfectas. Sostienen que Popper (1991) entiende que la ciencia y la filosofía son, en última instancia, sentido común esclarecido.

En el segundo caso, Fals Borda considera que es fundamental conocer y apreciar el papel que juegan el saber popular, el sentido común y la cultura popular para "obtener y crear conocimiento académico-científico" (1990:27). Para abonar esta idea cita los innumerables casos de fármacos utilizados por la medicina moderna que derivan de la medicina indígena.

La segunda opción entiende que la ciencia se construye contra el sentido común. Enriquez y Figueroa (2014) sostienen que autores como Bachelard, Bourdieu, Chamboredon y Passeron consideran que el "sentido común es ilusorio y superficial y, por ello, se constituye en un obstáculo epistemológico que ciega la mirada de los sujetos" (2014:46).

Estos autores citados proponen la "ruptura epistemológica como método de conquistar el conocimiento contra la ilusión del saber inmediato".

La tercera opción está representada por la propuesta de De Souza Santos, que habla de una "doble ruptura epistemológica". Se debe superar el sentido común "mediante la solidez científica y el rigor académico", y generar luego una segunda ruptura que procure la "construcción de un nuevo sentido común emancipador que transforme el conocimiento académico-científico y su potencial en un movimiento en espiral que lleve a un nivel superior al sentido común" (2006:30).

A este respecto, Souza Santos propone una "ecología de saberes", en la cual:

"No se trata de 'descredibilizar' las ciencias ni de un fundamentalismo esencialista 'anti-ciencia'; nosotros, como científicos sociales, no podemos hacer eso. Lo que vamos a intentar hacer es un uso contra-hegemónico de la ciencia hegemónica. O sea, la posibilidad de que la ciencia entre no como monocultura sino como parte de una ecología más amplia de saberes, donde el saber científico pueda dialogar con el saber laico, con el saber popular, con el saber de los indígenas, con el saber de las poblaciones urbanas marginales, con el saber campesino. Esto no significa que todo vale lo mismo. Lo discutiremos con el tiempo. Estamos en contra de las jerarquías abstractas de conocimientos, de las monoculturas que dicen, por principio, 'la ciencia es la única, no hay otros saberes'. Vamos a partir, en esta ecología, de afirmar que lo importante no es ver cómo el conocimiento representa lo real, sino conocer lo que un determinado conocimiento produce en la realidad; la intervención en lo real. Estamos intentando una concepción pragmática del saber. ¿Por qué? Porque es importante saber cuál es el tipo de intervención que el saber produce. No hay duda de que para llevar al hombre o a la mujer a la luna no hay conocimiento mejor que el científico; el problema es que también sabemos hoy que para preservar la biodiversidad, de nada sirve la ciencia moderna. Al contrario, la destruye. Porque lo que ha conservado y mantenido la biodiversidad son los conocimientos indígenas y campesinos. ¿Es acaso una coincidencia que el $80 \%$ de la biodiversidad se encuentre en territorios indígenas? No. Es porque la naturaleza allí es la Pachamama, no es un recurso natural: 'es parte de nuestra sociabilidad, es parte de nuestra vida'; es un pensamiento antidicotómico. Entonces lo que tengo que evaluar es si se va a la luna, pero también si se preserva la biodiversidad. Si queremos las dos cosas, tenemos que entender que necesitamos de dos tipos de conocimiento y no simplemente de uno de ellos. Es realmente un saber ecológico el que estoy proponiendo". (2006:21)

A nuestro criterio, la propuesta de Souza Santos es una alternativa interesante a poner en práctica, sabiendo además que coincide con la propuesta de Paulo Freire que sugiere en varios pasajes de su obra la posibilidad de partir del sentido común para construir un saber nuevo de transformación en la medida en que exista un espacio de diálogo y mutua interpelación de saberes. 


\section{El desarrollo de los Espacios de Formación Integral y sus posibilidades}

Luego de varios años de trabajo en la construcción de la integralidad y específicamente en el desarrollo de los Espacios de Formación Integral (EFI) en las distintas facultades y escuelas, debemos comenzar a reflexionar sobre sus potencialidades, dificultades y desafíos.

Los EFI se constituyen por múltiples lugares del proceso educativo universitario, como el espacio áulico curricular, las pasantías, espacios de formación preprofesional, cursos optativos, etc., en donde se conjuga y articula la extensión y la investigación al proceso de enseñanza y aprendizaje. De todas formas en la UdelaR hemos insistido y también avanzado en la construcción de espacios integrales en el marco de las disciplinas o materias que son obligatorias, curriculares y, en consecuencia, acreditadas. Las potencialidades se vinculan con formaciones estudiantiles diferenciales en un sentido superador de las prácticas áulicas prevalecientes en la UdelaR. El estudio realizado por Romano y otros (2014), pone en evidencia algunas cuestiones centrales a la hora entender los efectos que provoca en los estudiantes su participación en los EFI. Rescatando de este estudio algunas de las principales percepciones estudiantiles, el siguiente gráfico pone en evidencia un proceso significativamente diferente.

Los resultados expresados en el gráfico dan cuenta de algunas cuestiones importantes del relacionamiento docentes/estudiantes, al conocimiento de realidades que el estudiante desde una formación exclusivamente áulica no conoce, a conocimientos de espacios interdisciplinares y multiprofesionales y al acercamiento a otras formas de saber o tipo de conocimiento no exclusivamente académico o científico. Estas percepciones encontradas en el estudio permiten sustentar que los EFI reinauguran procesos impulsados desde hace mucho tiempo en la UdelaR. Estos procesos han sido propuestos desde hace larga data por varios proyectos universitarios, de los que destacamos el Programa Aprendizaje y Extensión (APEX) pero también los procesos vinculados a las Plantas Piloto que la UdelaR desarrolló en la década del '50 (Bralich, 2006).

Las potencialidades se expresan también en la tarea docente. Los docentes que han participado de la experiencia ponen de manifiesto que esta forma de enseñar tiene la potencia de la enseñanza desde la problemática en concreto que se encuentra en el medio y consecuentemente da condiciones para aprendizajes significativos desde la realidad y no solo desde la "mediación áulica".

Estas potencialidades tienen su contracara: este tipo de actividad es más exigente en cuanto a los tiempos y compromisos de los estudiantes y los docentes. Implica tiempo de campo y no sólo tiempo de aula. Este tiempo combinado en general es mayor que el tiempo de las disciplinas que se dan exclusivamente en el aula. Además, en algunos casos implica que sea necesario convivir con situaciones sociales de vulnerabilidad que muchas veces son rechazadas como ámbitos de aprendizaje. Considerar este tema es absolutamente necesario ya que nos cuestiona, por ejemplo, la obligatoriedad de las prácticas integrales vinculadas a sectores subalternos de la sociedad, sobre todo para aquellos estudiantes y docentes que ideológicamente rechazan la vinculación y compromiso con estos sectores. Debemos ser extremadamente cuidadosos de no propiciar situaciones de no escucha o de invasión cultural por parte de estudiantes obligados a realizar trabajos en ámbitos que no son objeto de su preocupación y mucho menos de compromiso social.

La formación docente para esta tarea integral es otra de las cuestiones claves a considerar. Es absolutamente necesaria la formación para la enseñanza a nivel disciplinar, pero resulta imprescindible cuando a la tarea docente se agrega la enseñanza

Percepción acerca de los "logros" del EFI en los estudiantes

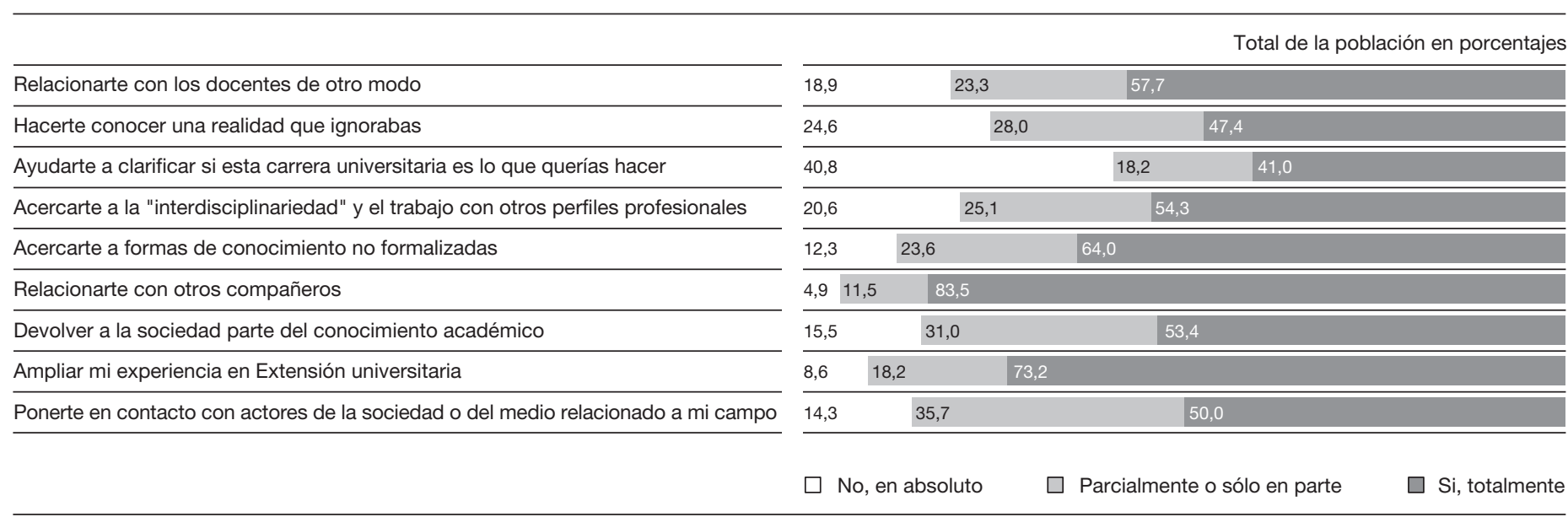

Fuente: elaboración propia en base a datos de la encuesta a estudiantes. 2013 


\section{6}

es absolutamente necesaria la formación

para la enseñanza a nivel disciplinar,

pero resulta imprescindible cuando a la

tarea docente se agrega la enseñanza

de la extensión y la investigación

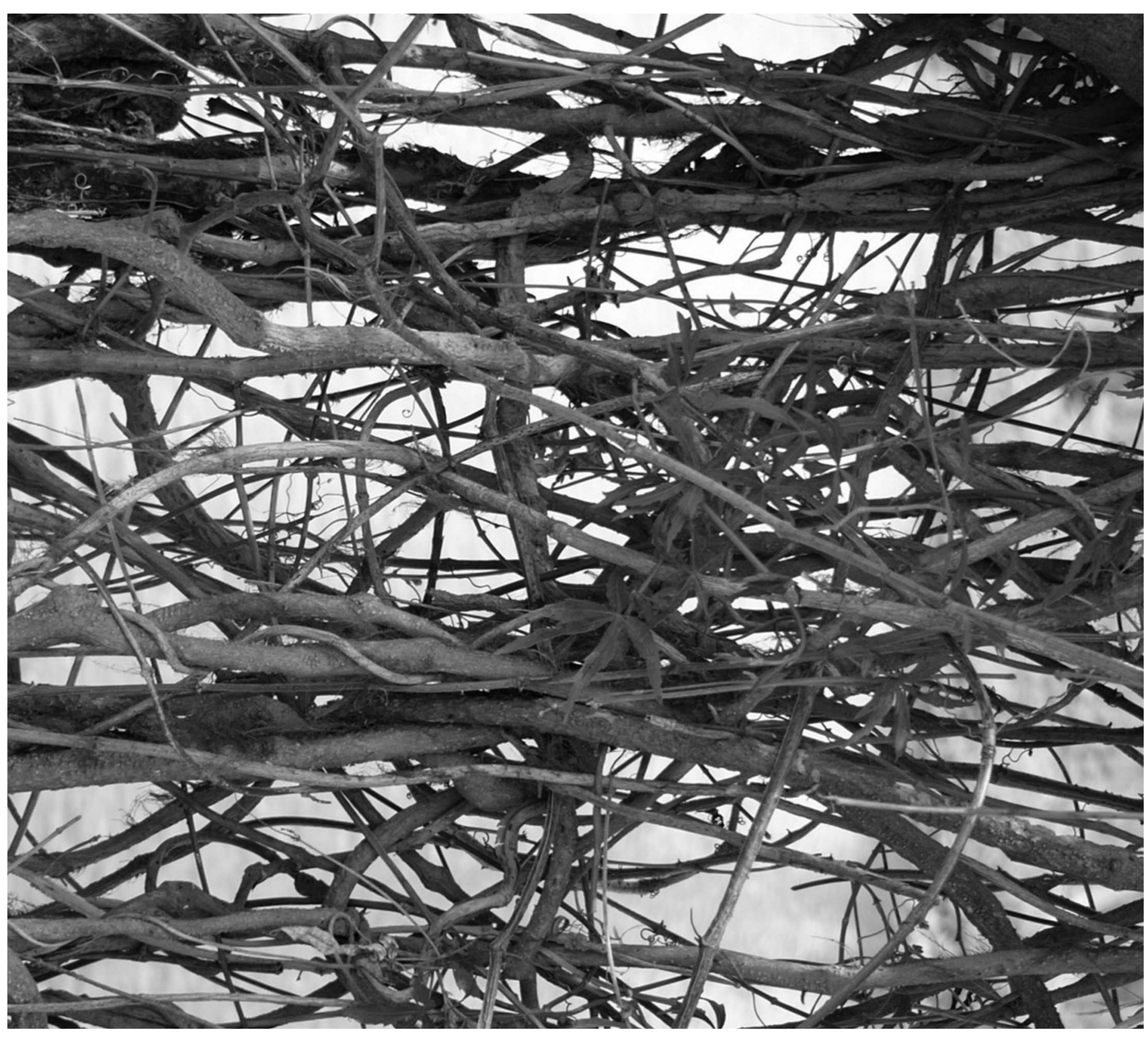

(C) Laura Malachesky 
de la extensión y la investigación. Procesos de acompañamiento teórico práctico para los docentes que se comienzan a vincular a estas prácticas son claves e imprescindibles.

Por último, luego de varios años de vinculación y apoyo a los procesos de integralidad, creemos condición sine qua non su impulso y consolidación a través de los Programas Integrales. En los últimos 25 años la UdelaR ha gestado el APEX, el Programa Integral Metropolitano (PIM), los Centros de Formación Popular y los Programas Integrales Temáticos. Estas son instancias territoriales y temáticas de vinculación permanente con la sociedad que permiten un vínculo estable y sistemático, que no está sujeto a los tiempos curriculares de la Universidad. Se componen de equipos docentes interdisciplinares que construyen demanda en forma permanente con los actores sociales con los que se vinculan, articulando esta demanda con los diferentes espacios universitarios (facultades, centros, escuelas).

El trabajo y desarrollo de estos programas ha puesto en evidencia la potencia que tienen a la hora de impulsar los procesos de integralidad. Deben ser priorizados si se pretende profundizar el desarrollo de las prácticas integrales. Ponen en evidencia que la contradicción entre programas centrales y desarrollo de la extensión e integralidad en la periferia o "descentralizada" (servicios universitarios) es una falacia que se sustenta en la ignorancia o desconocimiento intencional de la realidad.

La Universidad necesita seguir avanzado en el proceso de generar políticas de cara a la integralidad, el proceso desarrollado hasta el momento ha permitido a nuestro entender desencadenar corrimientos en las actividades de enseñanza y acercar a servicios más alejados de la extensión. La extensión se ha integrado como una función, tiene lugar en los curriculum a través de los nuevos planes de estudio de muchas facultades, se viene produciendo una "naturalización de la extensión, entendida como el propósito de que hacer extensión en la universidad llegue a ser tan "natural" y esperable como hacer enseñanza e investigación" (Arocena y Tommasino, 2011). Confiemos en que estos procesos que se han desarrollado puedan continuar y profundizarse a través de las prácticas y políticas universitarias.

\section{Algunas consideraciones finales}

Este artículo describe y analiza aspectos del proceso de integralidad de la práctica educativa llevado adelante desde la concepción de Segunda Reforma Universitaria puesta en marcha desde 2006 hasta 2014 en la UdelaR. Aborda algunas cuestiones teóricas y prácticas, evidencia la potencialidad que tiene para repensar y reorganizar la educación universitaria, además de la factibilidad de que la integralidad de funciones sea una de las llaves de transformación de la universidad contemporánea. Si bien este proceso no es novedoso si pensamos en su formulación, su puesta en juego en los últimos años, no solo en la UdelaR sino también en varias universidades de la región, como Universidad Nacional de Rosario, del Litoral, Entre Ríos, Mar del Plata, Cuyo, etc., debemos considerar la posibilidad de que la ampliación de las matriculas con la creación de nuevas universidades públicas se acompañe con el rediseño de las formas de aprender y enseñar. La incorporación de la extensión crítica en los procesos educativos jaquea los procesos estereotipados de las metodologías áulicas e inaugura la posibilidad de pensar la formación de universitarios y no solo técnicos formados de modo estrictamente disciplinar.

\section{Referencias bibliográficas}

Arocena, R. y Tommasino, H. (2011). Lineamientos generales para el avance de la curricularización de la extensión y generalización de las prácticas integrales en la Universidad de la República. Montevideo: Universidad de la República.

Bordoli, E. (2006). Aportes para la conceptualización de los Programas Integrales en la UdelaR. CD Rom. Montevideo: CSEAM.

Bralich, J. (2006). La extensión universitaria en el Uruguay. Universidad de la República. Comisión Sectorial de Extensión y Actividades en el Medio. Servicio Central de Extensión y Actividades en el Medio, Montevideo.

Enriquez, P.; Figueroa, P. (2014). Escuela de sectores populares. Notas para pensar la construcción de contenidos escolares desde el saber popular y el académico-científico. Buenos Aires: Miño y Davila.

Fals Borda, O, (1990). El problema de cómo investigar la realidad para transformarla por la praxis. Bogota: Tercer Mundo Editores.

Freire, P. (1983). Extensão ou comunicação? Rio de Janeiro: Paz e Terra.

Freire, P. (1996). Política y educación, México: Siglo XIX Editores.

Gatti, E. (2000). Los modelos pedagógicos en la educación superior. Temas y

Propuestas, (18), 24-43. Buenos Aires.

Pistrak, M. (2009). A Escola Comuna. San Pablo: Editora Expresao Popular.

Popper, K. (1991). La lógica de la investigación científica. México: Rei.

Romano, A. y otros (2014). Evaluación sobre las Políticas de Extensión relacionadas con los procesos de integralidad en la Universidad de la República. El caso de los Espacios de Formación Integral. Informe preliminar de resultados de la fase de encuesta a estudiantes. Montevideo: UdelaR.

Souza Santos, B. (2006). Renovar la teoría crítica y Reinventar la emancipación social [Encuentros en Buenos Aires]. CLACSO Libros.

Tommasino, H. y otros (2010). De la extensión a las prácticas integrales, en la reforma universitaria. La extensión en la renovación de la enseñanza. Espacios de formación integral. Montevideo: UdelaR.

Tommasino, H. y Cano, A. (2016a). Modelos de extensión universitaria en las universidades latinoamericanas en el siglo XXI: tendencias y controversias. Universidades, LXVI, Nueva época, (67, enero-marzo). México: UDUAL.

Tommasino, H. y Cano, A. (2016 b). Avances y retrocesos de la extensión critica en la Universidad de la República de Uruguay. Masquedós, 1(1), 9-23. Secretaría de Extensión UNICEN, Tandil, Buenos Aires.

UdelaR (2010). Hacia la reforma universitaria. La extensión en la renovación de la enseñanza. Espacios de formación integral. Montevideo: UdelaR.

UdelaR (30 de agosto de 2011). Ordenanza de estudios de grado. Consejo Directivo Central. Montevideo: UdelaR. 